\title{
ELECTRON MICROSCOPY OF HUMAN VAGINAL EPITHELIUM UNDER NORMAL AND EXPERIMENTAL CONDITIONS
}

\author{
ELSA B. SCHUCHNER, A. FOIX, G. A. BORENSTEIN \\ AND G. MARCHESE \\ Centro de Investigaciones en Reproducción, \\ Facultad de Medicina, Universidad de Buenos Aires, and \\ Servicio de Ginecologia, Hospital Argerich, Buenos Aires, Argentina
}

(Received 13th August 1973)

Recent studies have demonstrated that several types of intercellular connections maintain the architecture of the human epithelium (Karrer, 1960; Burgos \& Vargas Linares, 1970). Several types of cell junctions were also recognized between the vaginal epithelial cells of a number of species: guinea-pig (Burgos \& Wislocki, 1958), hamster (Vargas Linares \& Burgos, 1965; Vargas Linares, 1968), rat (Schuchner, Paczy \& Asch, 1972) and human (Vargas Linares \& Burgos, 1968; Burgos \& Vargas Linares, 1970).

The present study was designed to show the ultrastructural changes of the human vaginal epithelium under the influence of the local chelating action of EDTA and the subsequent recovery of the epithelium.

The biopsies were performed on clinically normal vaginal mucosae. Three biopsies were obtained from each of the eight women studied. The first biopsy was taken as the control for the other two. Immediately after the biopsy, a vaginal tampon impregnated with a solution of 0.2 м-EDTA ( $\mathrm{pH} \mathrm{3.5)} \mathrm{was}$ introduced in the vagina; the tampon was changed three times a day over a 3- or 6-day period. On the 4th or 7th day, the second biopsy was obtained. One month after the initial day of treatment, the third biopsy was taken.

The biopsies were removed under local anaesthesia from the upper portion of the vagina. After removal, the tissues were cut into small pieces and immediately fixed in a solution of glutaraldehyde $2.5 \%$ in phosphate buffer $(\mathrm{pH} \mathrm{6.5)}$ and postfixed in Caufield's solution.

The specimens were dehydrated in graded alcohol solutions and then embedded in Maraglas epoxy resin. Thin sections were cut on a Porter Blum ultramicrotome, mounted on formvar-coated grids, stained with uranyl acetate and lead citrate and examined in a Siemens Elmiskop I electron microscope.

Our observations were mainly focused on the stratum Malphighii. The cell junctions were seen to be present during the entire cycle, and reached maximal development at the end of the follicular phase. Three types of junctions were easily recognized: desmosomes, tight junctions and intermediate junctions. 
In the control biopsies (P1. 1, Figs 1 and 5), the cells of the stratum Malphighii were characterized by their irregular shape, large spherical nuclei and prominent nucleoli. The cytoplasm contained few organelles, tight junctions (belt-like structures encircling the cell) and desmosomes (discontinuous buttonlike structures consisting of two dense plaques on the opposing cell surfaces) with numerous tonofibrils that attached the cells to one another.

The effect of the EDTA-impregnated tampon could be described in several separate stages: the initial damage (after 3 days of treatment) included cytoplasmic damage in the perinuclear area, with different degrees of vacuolization in areas of cytoplasm not attached to the nuclear membrane (Pl. 1, Fig. 2).

After 6 days (Pl. 1, Figs 3, 4 and 6) the lesion was severe and the intercellular junctions had lost their structure, a wide space appeared between the interdigitations; the tonofilaments that belonged to the desmosomes were separated from the cytoplasmic membrane. The nucleus of these cells had shrunk and their dense chromatin was distributed in patches, indicating severe cytological damage.

The biopsies taken 1 month later showed complete replacement of the cells, even after 6 days of exposure to EDTA.

The desmosomal junctions in the vaginal epithelium may act either as a barrier to the passage of material or cells (leucocytes) from the lumen to the epithelium, or as sites of maximum interchange between the cells.

The remarkable effect of the EDTA-chelating treatment was the opening of the desmosomes and increase in size of the intercellular spaces; this was probably due to depletion of calcium.

Vargas Linares \& Burgos (1965) showed that the desmosomes opened during the passage of leucocytes through the vaginal epithelium at metoestrus, and Moscona (1961) pointed out that calcium is an important element in the maintenance of cellular junctions.

Vargas Linares \& Burgos (1965) also described the effect of perfusion of hamster epithelium with $0 \cdot 2 \mathrm{M}$-EDTA. There was a loss of density and definition

\section{EXPLANATION OF PLATE 1}

FIG. 1. Micrograph showing a normal vaginal biopsy. The cell of the Malphighian epithelium has an irregular shape, a big round nucleus and a prominent reticular type of nucleolus. The cytoplasm contains numerous tonofilaments. Several desmosomes could be seen among the cells. $n$ : nucleus, $c$ : cytoplasm. $\times 3500$.

FIc. 2. Micrograph showing a biopsy of the vaginal epithelium after 3 days of exposure to EDTA. The cytoplasm of the perinuclear area has started to vacuolize even though the nucleus looks normal. $n$ : nucleus, $c$ : cytoplasm. $\times 4200$.

Fig. 3. Micrograph showing a biopsy of the vaginal epithelium after 6 days of exposure to EDTA. The nucleus as well as the cytoplasm appears severely damaged, the chromatin is condensed and few cytoplasmic organelles could be seen. The intercellular junctions are no longer present; clear spaces could be seen between the cells. $n$ : nucleus, c: cytoplasm. $\times 2800$.

Fig. 4. Higher magnification of the nuclear area of Fig. 3. $n$ : nucleus, c: cytoplasm. $\times 7000$.

Fig. 5. Micrograph showing desmosomes (arrow) between epithelial cells from an untreated vagina. $\times 14,000$.

FIG. 6. Micrograph showing similar desmosomal area (arrow) from the epithelium of a vagina exposed to EDTA. $\times 14,000$. 
PLATE 1
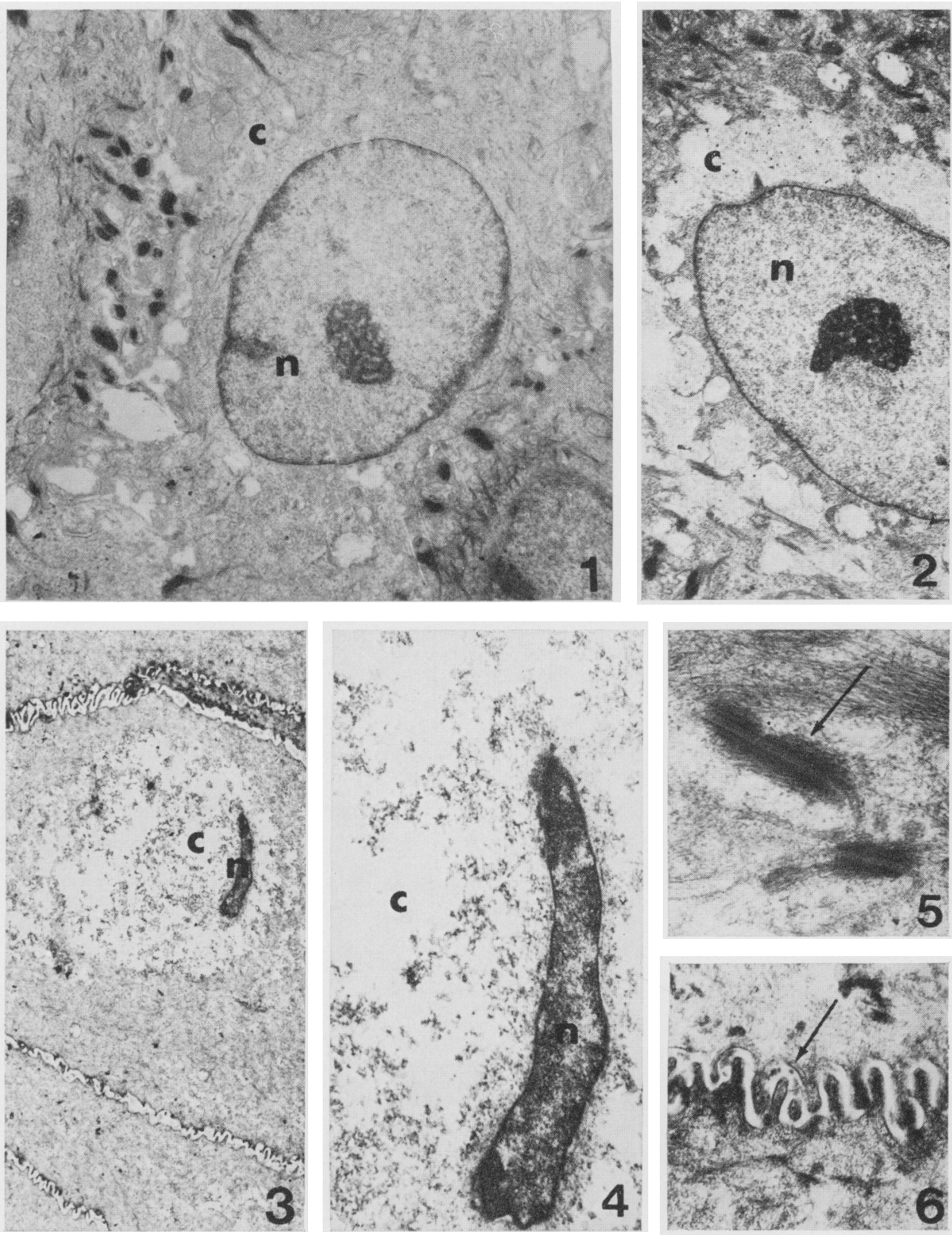
of the desmosomes, expansion of the intercellular space and splitting with evident separation of the cells.

These observations of the effect of local application of EDTA were similar to the event described by Vargas Linares \& Burgos (1968) during leucocyte invasion, being in both cases probably a sequestrating action of calcium performed either by the chelating chemical mediator or by the white blood cells directly on vaginal desmosomes. In both cases, the effect was reversible.

The present observations have shown the reversibility of the effects of local treatment with EDTA. This treatment probably resulted in a change in the vaginal $\mathrm{pH}$ which could be of a great use in the sperm-vaginal relationship, especially since the effect is reversible.

This work was supported in part by a grant from The Population Council Inc., New York, to one of us (E.B.S.).

The authors are much obliged to Miss Alicia Paczy and Miss Myrna Mortyrú for their technical assistance.

\section{REFERENGES}

Burgos, M. H. \& Vargas Linares, C. (1970) Cell junctions in the human vaginal epithelium. Am. $\mathcal{F}$. Obstet. Gynec. 108, 565.

Burgos, M. H. \& Wislocki, G .B. (1958) The cyclical changes in the mucosa of the guinea pig's uterus, cervix and vagina and in the sexual skin, investigated by the electron microscope. Endocrinology, 63, 106.

KARRER, H. E. (1960) Cell interconnections in normal human cervical epithelium. F. biophys. biochem. Cytol. 7, 181.

Moscona, A. A. (1961) How cells associate. Scient. Am. 205, 143.

Schuchner, E. B., PACzy, A. \& Asch, R. (1972) Subcellular distribution of some inorganic cations in the vaginal epithelium of the rat. F. Reprod. Fert. 30, 283.

Vargas Linares, C. (1968) Plasma cells in the hamster vagina: cyclical and experimental variations. 7. Reprod. Fert. 15, 389.

VARgAs Linares, G. \& BuRgos, M. H. (1965) Junctional complexes of the hamster vagina, under normal and experimental conditions. Q. Jl exp. Physiol. 50, 481.

Vargas Linares, G. \& Burgos, M. H. (1968) Migrations of lymphocytes in the normal human vagina. Am. F. Obstet. Gynec. 102, 1094. 